\title{
Vorwort zur 4. Auflage
}

Seit der 3. Auflage dieses Buches sind fünf Jahre vergangen. Seitdem sind eine Fülle von Entscheidungen ergangen und zahlreiche Literaturbeiträge erschienen, die jetzt in der 4. Auflage berücksichtigt werden.

Das Werk ist vor allem an Praktiker gerichtet, die in Ihrer Arbeit mit dem Handelsregister zu tun haben. Zur Arbeit in der Praxis dienen die zahlreichen Musterformulare, die aktualisiert und ergänzt wurden, zum Beispiel zum Genehmigten Kapital bei der GmbH. Eine Garantie, dass diese Musterformulare von allen Registergerichten in der Bundesrepublik akzeptiert werden, kann verständlicherweise nicht übernommen werden. Es kann aber versprochen werden, dass die Musterformulare nach besten Wissen und Gewissen gefertigt wurden.

Gesetzgebung und Literatur sind bis zum 6. Juli 2019 berücksichtigt. Die Autoren sind wie immer - für Hinweise und Anregungen zur Optimierung dieses Buchs dankbar.

Berlin, Oktober 2019

Peter Ries

Rita Bauer

Sven Rudolph

Christian Schulte 
